\title{
Veno-venous extracorporeal membrane oxygenation in coronavirus disease 2019: a case series
}

Joe Zhang $\oplus^{1}$, Blair Merrick², Genex L. Correa', Luigi Camporota', Andrew Retter ${ }^{1}$, Andrew Doyle $\mathbb{\circledR}^{3}$, Guy W. Glover ${ }^{1}$, Peter B. Sherren ${ }^{1}$, Stephen J. Tricklebank ${ }^{1}$, Sangita Agarwal ${ }^{4}$, Boris E. Lams ${ }^{5}$, Nicholas A. Barrett ${ }^{1}$, Nicholas loannou', Jonathan Edgeworth ${ }^{2}$ and Christopher I.S. Meadows ${ }^{1}$

Affiliations: ${ }^{1}$ Dept of Critical Care Medicine, Guy's and St Thomas' NHS Foundation Trust, London, UK. ${ }^{2}$ Dept of Infectious Diseases, Guy's and St Thomas' NHS Foundation Trust, London, UK. ${ }^{3}$ Dept of Haematology, Guy's and St Thomas' NHS Foundation Trust, London, UK. 'Dept of Rheumatology, Guy's and St Thomas' NHS Foundation Trust, London, UK. ${ }^{5}$ Dept of Respiratory Medicine, Guy's and St Thomas' NHS Foundation Trust, London, UK.

Correspondence: Christopher I.S. Meadows, Dept of Critical Care Medicine, Guy's and St Thomas' NHS Foundation Trust, Westminster Bridge Rd, London, SE1 7EH, UK. E-mail: chris.meadows@agstt.nhs.uk

\section{ABSTRACT}

Background: The use of veno-venous extracorporeal membrane oxygenation (VV-ECMO) in severe hypoxaemic respiratory failure from coronavirus disease 2019 (COVID-19) has been described, but reported utilisation and outcomes are variable, and detailed information on patient characteristics is lacking. We aim to report clinical characteristics, management and outcomes of COVID-19 patients requiring VV-ECMO, admitted over 2 months to a high-volume centre in the UK.

Methods: Patient information, including baseline characteristics and clinical parameters, was collected retrospectively from electronic health records for COVID-19 VV-ECMO admissions between 3 March and 2 May 2020. Clinical management is described. Data are reported for survivors and nonsurvivors.

Results: We describe 43 consecutive patients with COVID-19 who received VV-ECMO. Median age was 46 years (interquartile range $35.5-52.5$ ) and $76.7 \%$ were male. Median time from symptom onset to VVECMO was 14 days (interquartile range 11-17.5). All patients underwent computed tomography imaging, revealing extensive pulmonary consolidation in $95.3 \%$, and pulmonary embolus in $27.9 \%$. Overall, $79.1 \%$ received immunomodulation with methylprednisolone for persistent maladaptive hyperinflammatory state. Vasopressors were used in $86 \%$, and $44.2 \%$ received renal replacement therapy. Median duration on VVECMO was 13 days (interquartile range 8-20). 14 patients died (32.6\%) and 29 survived (67.4\%) to hospital discharge. Nonsurvivors had significantly higher d-dimer $\left(38.2\right.$ versus $9.5 \mathrm{mg} \cdot \mathrm{L}^{-1}$, fibrinogen equivalent units; $\mathrm{p}=0.035)$ and creatinine ( 169 versus $73 \mu \mathrm{mol} \cdot \mathrm{L}^{-1} ; \mathrm{p}=0.022$ ) at commencement of VV-ECMO. Conclusions: Our data support the use of VV-ECMO in selected COVID-19 patients. The cohort was characterised by high degree of alveolar consolidation, systemic inflammation and intravascular thrombosis.

@ERSpublications

VV-ECMO, when offered to \#COVID19 patients in refractory respiratory failure, can be associated with favourable outcomes. This is a detailed case series of 43 COVID-19 patients requiring VV-ECMO from a UK centre. $67.4 \%$ survived to hospital discharge. https://bit.ly/3ko9Ucu

Cite this article as: Zhang J, Merrick B, Correa GL, et al. Veno-venous extracorporeal membrane oxygenation in coronavirus disease 2019: a case series. ERJ Open Res 2020; 6: 00463-2020 [https:// doi.org/10.1183/23120541.00463-2020]. 


\section{Introduction}

A significant cohort of patients with coronavirus disease 2019 (COVID-19) go on to develop severe respiratory failure, requiring critical care admission. Reports have described the use of veno-venous extracorporeal membrane oxygenation (VV-ECMO) in a subset of critically ill patients, with utilisation ranging from $11 \%$ to $32 \%$ [1-3]. VV-ECMO is indicated for patients with potentially reversible, refractory, life-threatening hypoxaemia or hypercapnia or in patients where acceptable oxygenation or decarboxylation can be obtained only with injurious ventilatory settings. While VV-ECMO was associated with improved outcome during the H1N1 influenza pandemic [4, 5], COVID-19 demonstrates features unique from other respiratory infections and early case series have reported high mortality in patients on ECMO [6-8].

Given the lack of detailed information about patient characteristics and their clinical course, balanced with the need for judicious use of resources in the context of a pandemic, it is important to understand the role of VV-ECMO in COVID-19. We aim to describe, in detail, the clinical characteristics, management and outcomes of COVID-19 VV-ECMO patients from a high-volume UK ECMO centre, over a 2-month period of the pandemic.

\section{Methods}

Case selection

All COVID-19 patients admitted for VV-ECMO to Guy's and St Thomas' NHS Foundation Trust (GSTFT) in London, over a 2-month period (3 March 2020 to 2 May 2020) covering the peak of the pandemic, are included. Suitability for VV-ECMO was assessed in line with UK national commissioning criteria [9], requiring a lung injury (Murray) score $\geqslant 3$ [10], or uncompensated hypercapnic acidosis with $\mathrm{pH}<7.2$. National criteria were adapted for the COVID-19 pandemic on 10 April 2020 [11] to include clinical frailty scale $\leqslant 3$ [12], the use of the Respiratory ECMO Survival Prediction (RESP) score [13] to aid pre-ECMO decision-making (with RESP score $\leqslant 3$ requiring agreement between at least two centres), and an exclusion of "refractory multi-organ failure". At the time of this series, detection of SARS-CoV-2 RNA on nose and throat swabs or bronchoalveolar lavage (BAL) using multiplexed-tandem PCR technology for detection of two gene targets, ORF 1a and ORF 8 (AusDiagnostics, Mascot, Australia), remained the gold standard. All patients, at point of referral, had either laboratory-confirmed or clinically suspected COVID-19 pneumonia; four patients without a positive result at time of referral subsequently tested positive from admission samples at GSTFT.

\section{Patient clinical pathway}

GSTFT is a national VV-ECMO centre commissioned to provide regional ECMO retrieval and provision [9]. At the start of the pandemic, GSTFT ECMO capacity was doubled through adaptation of each bedspace to accommodate two patients on ECMO. Patients were retrieved from referring hospitals via a previously described standard pathway [14], with no deviation in practice, aside from use of recommended personal protective equipment. Standard GSTFT practice is bifemoral percutaneous cannulation at the referring hospital, and use of Maquet Cardiohelp (Maquet, Rastatt, Germany) consoles. Following retrieval, all patients underwent computed tomography (CT) imaging of head, thorax (including CT pulmonary angiogram), abdomen and pelvis. Lung recruitment CT imaging at ventilator pressures of $5 \mathrm{cmH}_{2} \mathrm{O}$ and $45 \mathrm{cmH}_{2} \mathrm{O}$ were performed, unless pneumothorax was detected on initial scan or pulmonary air leak was suspected, to assess lung recruitment potential and delineate underlying lung parenchyma [15]. Diagnostic bronchoscopy and BAL for bacterial culture, viral and SARS-CoV-2 PCR was performed on all patients within the first $24 \mathrm{~h}$. Patients without haemorrhagic complications were anticoagulated with unfractionated heparin infusion, targeting anti-Xa levels $\left(0.3-0.7 \mathrm{UI} \cdot \mathrm{mL}^{-1}\right)$.

Patients were ventilated with protective lung parameters. Mechanical ventilation was generally initiated using standardised settings: positive end-expiratory pressure (PEEP) 10-15 $\mathrm{cmH}_{2} \mathrm{O}$, tidal volume $2-4 \mathrm{~mL} \cdot \mathrm{kg}^{-1}$ of predicted body weight provided that driving pressure (plateau minus PEEP total) could be kept at $10 \mathrm{cmH}_{2} \mathrm{O}$, and plateau pressure $<25 \mathrm{cmH}_{2} \mathrm{O}$. Initial respiratory rate was generally maintained at 10 breaths $\min ^{-1}$ to limit overall mechanical power [15]. In patients with high potential for lung recruitment, as demonstrated on low/high pressure CT imaging, higher PEEP (near $15 \mathrm{cmH}_{2} \mathrm{O}$ ) or time-controlled adaptive ventilation was used, with mean airway pressures of $23-26 \mathrm{cmH}_{2} \mathrm{O}$ depending on body mass index and small airway closures (following assessment of a low-flow pressure-volume loop). Inspiratory oxygen fraction $\left(F_{\mathrm{IO}_{2}}\right)$ on the ventilator was kept at 30-40\%, and ECMO support titrated to achieve arterial oxygen tension $\left(P_{\mathrm{aO}_{2}}\right)>60 \mathrm{mmHg}$ and $\mathrm{pH}$ 7.35-7.40.

Patients received a course of broad-spectrum antibiotics on arrival, targeted to known microbiology where possible. A subset of patients with failure to progress and signs of a sustained hyperinflammatory state (fevers, persistently elevated C-reactive protein and/or ferritin, sustained organ dysfunction and 
hypoxaemia), in the absence of untreated active infection (bacteria or fungal species detected on blood culture and BAL, low procalcitonin and galactomannan), were treated with low-dose methylprednisolone regimens of $1-2 \mathrm{mg} \cdot \mathrm{kg}^{-1} \cdot \mathrm{day}^{-1}$ for 5-7 days, with halving in dose every 5-7 days, similar to published protocol [16]. This dosing regimen was chosen for its relative safety profile [17, 18]. Patients with persistent hyperinflammatory disease behaviour despite corticosteroids, or those with an "H score" greater than 169 suggesting secondary haemophagocytic lymphohistiocytosis [19, 20] were considered for treatment with the interleukin (IL)-1 receptor antagonist anakinra [21, 22]. Patients with persistent hypoxaemia and radiological abnormality despite low-dose corticosteroids, or patients who demonstrated early fibrosis on CT, were treated with high dose "pulsed" methylprednisolone at doses of $1 \mathrm{~g}$ for 3 days, followed by $1 \mathrm{mg} \cdot \mathrm{kg}^{-1}$ per day, followed by a weaning regimen. Treatments were given in consultation with local lung inflammation specialists.

Patients generally remained paralysed for an initial $24 \mathrm{~h}$, particularly if strong inspiratory efforts persisted despite adequate sedation, or if asynchronies due to deep sedation were noted (e.g. reverse triggering). Daily sedation wean was then undertaken in stable patients to maximise wakefulness. A specialist physiotherapy team assessed patients on a daily basis for both secretion clearance, and early rehabilitation. Ventilation weaning was based on daily assessment of lung mechanics, as well as ability to spontaneously ventilate without injurious tidal volumes, respiratory rate and inspiratory effort (including measurements of P0.1-100 ms airway occlusion pressure), that might contribute to patient self-inflicted lung injury. Criteria for decannulation from VV-ECMO in this cohort included maintained fractional inspired oxygen $<0.5$ and noninjurious ventilatory effort, with ECMO sweep gas turned off for at least $24 \mathrm{~h}$. The full protocol of weaning from VV-ECMO is described and available [23].

\section{Data collection and analysis}

Data were collected retrospectively from electronic records, including the IntelliVue Clinical Information Portfolio (Philips, Eindhoven, the Netherlands). Pre-ECMO data were obtained from ECMO referral systems [24], paper records or direct interview with members of retrieval teams. RESP score was calculated at the time of referral. Sequential Organ Failure Assessment score was calculated from pre-ECMO clinical parameters and day 0 ECMO laboratory results, with Glasgow Coma Scale presumed to be 15 unless deranged consciousness pre-intubation was confirmed. Data were collected until all patients had reached either death (nonsurvivors) or discharge from hospital (survivors). Quantitative variables are presented with median and interquartile range (IQR), and categorical variables are presented as frequencies and percentages. Missing data were not imputed and are recognised in tables by adjusted $n$-values. Comparison of nonparametric continuous variables used Mann-Whitney U-tests with significance at $\mathrm{p}<0.05$ (Python: package SciPy v.1.4.1). The study had institutional approval and waiver of individual informed consent (reference no.10796), qualifying as service evaluation defined by the UK National Health Service Health Research Authority (www.hra.nhs.uk).

\section{Results}

\section{Demographic and pre-ECMO characteristics}

Forty-three patients with COVID-19 were accepted and admitted for VV-ECMO based on the listed criteria, out of 215 patients referred to GSTFT during the study period. Patient characteristics are shown in table 1. Median age was 46 years (IQR 35.5-52.5), ranging from 26 to 66. Most patients were male (33, 76.7\%), 28 (65.1\%) patients came from a Black, Asian and Minority Ethnic background and (21, 48.8\%) were obese. Refractory and life-threatening hypoxia was an indication for VV-ECMO in all: median partial pressure of oxygen to fraction of inspired oxygen ratio $\left(P_{\mathrm{aO}_{2}} / F_{\mathrm{IO}_{2}}\right)$ was $67.5 \mathrm{mmHg}$ (IQR 58.9-77.8) at referral. $11(25.6 \%)$ patients additionally had uncompensated respiratory acidosis with $\mathrm{pH}<7.20$. Median static compliance was $30 \mathrm{~mL} \cdot \mathrm{cmH}_{2} \mathrm{O}^{-1}$ (IQR 21.5-33.6) in 24 patients with sufficient data for the calculation. Airway pressure release ventilation was used widely pre-ECMO (24 patients, 55.8\%). Forty (93\%) patients had undergone prone positioning prior to ECMO, and all patients had received neuromuscular blockade. Twenty-one (48.8\%) patients received vasopressors at referral, and $3(7 \%)$ required acute renal replacement therapy. Excluding one patient who acquired COVID-19 nosocomially, the median time from hospital admission to VV-ECMO was 7 (IQR 5-9) days. Median time from reported start of COVID-19 symptoms to VV-ECMO was 14 (IQR 11-18) days, and median from invasive ventilation to VV-ECMO was 5 (IQR 2-6) days.

\section{Clinical diagnostics and features}

The majority of patients $(41,95.3 \%)$ had extensive pulmonary consolidation on CT in either all lobes (figure 1a), or in a dependent distribution (figure 1b) with minimal sparing. Nineteen patients underwent lung recruitment imaging, 17 (89.5\%) showed at least moderate recruitability as assessed visually by a radiologist. Twelve (27.9\%) patients had pulmonary embolism (PE), five involving at least one main 
TABLE 1 Baseline and pre-extracorporeal membrane oxygenation (ECMO) characteristics

\begin{tabular}{|c|c|c|c|}
\hline & Survivors ( $\mathrm{n}=29$ ) & Nonsurvivors $(n=14)$ & Total (n=43) \\
\hline Age years & $45(35-49)$ & $52.5(43.8-53)$ & $46(35.5-52.5)$ \\
\hline \multicolumn{4}{|l|}{ Sex } \\
\hline Male & $20(69.0)$ & 13 (92.9) & 33 (76.7) \\
\hline BMI $\mathrm{kg} \cdot \mathrm{m}^{-2}$ & $29(28-34)$ & $31(26-34)$ & $29(27-34)$ \\
\hline \multicolumn{4}{|l|}{ Ethnicity (ISARIC) } \\
\hline South Asian & $11(37.9)$ & 2 (14.3) & $13(30.2)$ \\
\hline East Asian & $2(6.9)$ & $2(14.3)$ & 4 (9.3) \\
\hline Mixed & $0(0)$ & $1(7.1)$ & $1(2.3)$ \\
\hline \multicolumn{4}{|l|}{ Comorbidity } \\
\hline Obesity $\#$ & $14(48.3)$ & $7(50.0)$ & $21(48.8)$ \\
\hline Hypertension & $5(17.2)$ & $5(35.7)$ & 10 (23.3) \\
\hline$P_{\mathrm{aO}_{2}} / F_{\mathrm{IO}_{2}}{ }^{9}$ & $67.5(61.6-77.6)$ & $61.5(53.7-79.5)(n=13)$ & $67.5(58.9-77.8)(n=42)$ \\
\hline $\mathrm{pH}$ & $7.34(7.23-7.39)(n=28)$ & $7.25(7.17-7.30)(n=13)$ & $7.30(7.19-7.36)(n=41)$ \\
\hline$P_{\text {aco }}, \mathrm{mmHg}$ & $66.5(52.5 .0-70.5)$ & $69.8(60.3-78.4)$ & $67.5(53.1-75.8)$ \\
\hline Ventilation days before ECMO & $5(2-6)$ & $4(1-6)$ & $5(2-6)$ \\
\hline SOFA score & $6(4-8)$ & $9(6-12)$ & $7(4-10)$ \\
\hline
\end{tabular}

pulmonary artery. Five (11.6\%) additional patients demonstrated areas of pulmonary infarction without PE. Left ventricular impairment on admission echocardiography was rare, in one patient (2.3\%) with mild-to-moderate impairment only. In six (14\%) patients, right ventricular dysfunction was found concurrently with PE. Six (14\%) patients had small foci of subarachnoid haemorrhage on CT head, two of which demonstrated a local mass effect. Three (7\%) patients had ischaemic infarction. Patients on day 0 of VV-ECMO had severe lymphopenia (median $0.6 \times 10^{9} \cdot \mathrm{L}^{-1}$ (IQR $0.5-1.1$ ), elevated neutrophil to lymphocyte ratio (median 12.8 (IQR 9.2-22.5)), and high C-reactive protein (median $326 \mathrm{mg} \cdot \mathrm{L}^{-1}$ (IQR 245-368)), ferritin (median $1907 \mu \mathrm{g} \cdot \mathrm{L}^{-1}$ (IQR 1153-4083)), and d-dimer (median $11.7 \mathrm{mg} \cdot \mathrm{L}^{-1}$ fibrinogen equivalent units (IQR 6.4-41.7). Acute kidney injury (creatinine $\geqslant 105 \mu \mathrm{mol} \cdot \mathrm{L}^{-1}$ ) was a common feature (21 patients, $48.8 \%)$. Data are shown in table 2 .

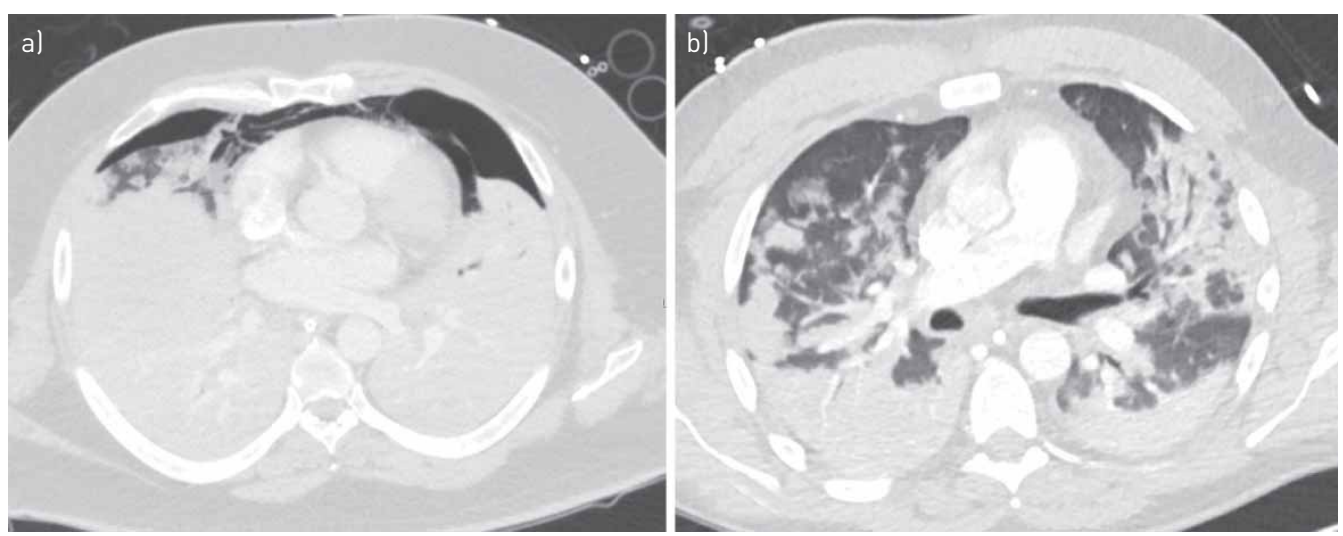

FIGURE 1 a) Almost completely consolidated lungs, with minor sparing the right middle lobe periphery where there is scattered ground-glass opacification. Bilateral small pneumothoraces and moderate pneumomediastinum. b) Dense consolidation involving most of the lower lobes, further areas of consolidation seen in all other lobes in a patchy distribution, with ground-glass opacification. 
TABLE 2 Extracorporeal membrane oxygenation (ECMO) admission investigation findings

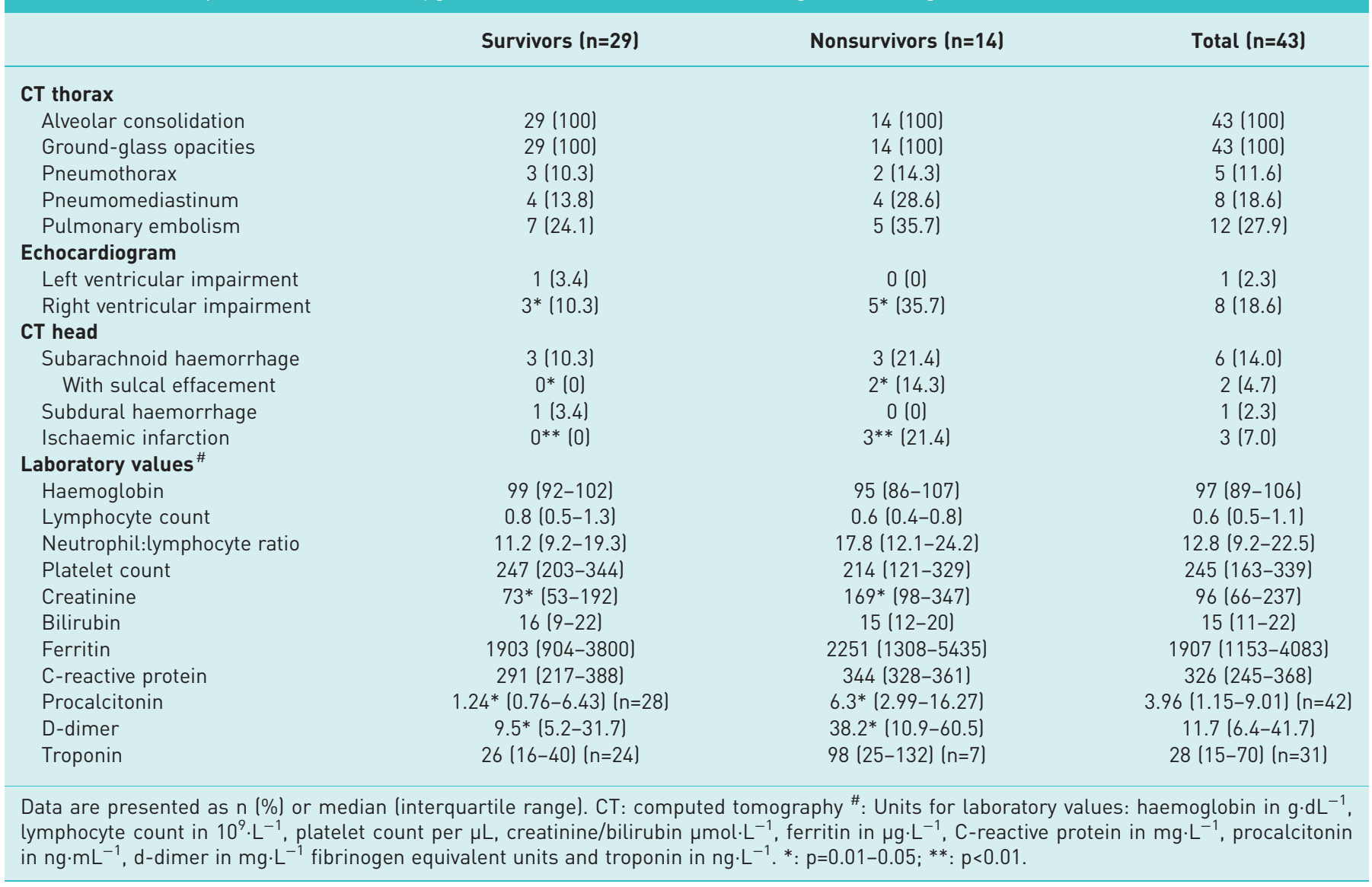

\section{ECMO course and outcomes}

Twenty-nine patients (67.4\%) were successfully decannulated from VV-ECMO and survived until hospital discharge. Twelve (27.9\%) patients died on ECMO, and two (4.7\%) died following decannulation. Causes of death and complications are summarised in table 3. Median days on ECMO was 14 (IQR 8-21) for survivors. Nonsurvivors had significantly greater procalcitonin $\left(6.30\right.$ versus $\left.1.24 \mathrm{ng} \cdot \mathrm{mL}^{-1} ; \mathrm{p}=0.028\right)$, d-dimer (38.2 versus $9.5 \mathrm{mg} \cdot \mathrm{L}^{-1}$ fibrinogen equivalent units; $\left.\mathrm{p}=0.035\right)$ and creatinine (169 versus $\left.73 \mu \mathrm{mol} \cdot \mathrm{L}^{-1} ; \mathrm{p}=0.022\right)$ at admission. Common complications were haemorrhage requiring $\geqslant 1$ packed red blood cell transfusion $(8,18.6 \%)$ including two bleeds after tracheostomy and two spontaneous retroperitoneal haemorrhages (one leading to death), and tension pneumothorax $(5,11.6 \%)$ that contributed to mortality in three patients. One patient developed myocarditis, with cardiac tamponade resulting in death. Nineteen (44.2\%) patients required renal replacement therapy and the majority $(86 \%)$ required noradrenaline at moderate doses during ECMO (peak dose median $0.19 \mu \mathrm{g} \cdot \mathrm{kg}^{-1} \cdot \mathrm{min}^{-1}$ (IQR 0.11-0.35)). Seventeen (39.5\%) patients required at least one circuit change during their ECMO for membrane thrombosis; mean ECMO days per each circuit change (numerator as sum of all days on ECMO of entire cohort) was 29.6. In 31 patients decannulated from ECMO, 17 (54.8\%) demonstrated cannula-related peripheral venous thrombosis on Doppler imaging. The majority of patients (79.1\%) received immunomodulation, typically methylprednisolone at $1-2 \mathrm{mg} \cdot \mathrm{kg}^{-1} \cdot \mathrm{day}^{-1}$ although 9 (20.9\%) received higher "pulsed" doses and 10 (23.3\%) received anakinra (table 4$)$.

\section{Microbiology}

During their period of ECMO support, 15 (34.9\%) patients developed bacterial respiratory infection with Klebsiella spp. (K. aerogenes (6), K. oxytoca (1), and K. pneumoniae (8)). In 2 patients this grew from BAL on day 0 of ECMO, whereas later infection was detected in 13 patients. The most frequent fungal isolate was Candida spp. (15 patients, 34.9\%), all light or scanty growth from BAL. There were four significant bloodstream infections, two with $K$. oxytoca, one with Proteus mirabilis and a persistent vancomycin-resistant Enterococcus secondary to a deep-seated focus (retroperitoneal haematoma). Aspergillus fumigatus grew from BAL in two patients, and two others developed cytomegalovirus viraemia with IgG positivity. 


\section{TABLE 3 Time course, causes of death and complications on extracorporeal membrane oxygenation (ECMO)}

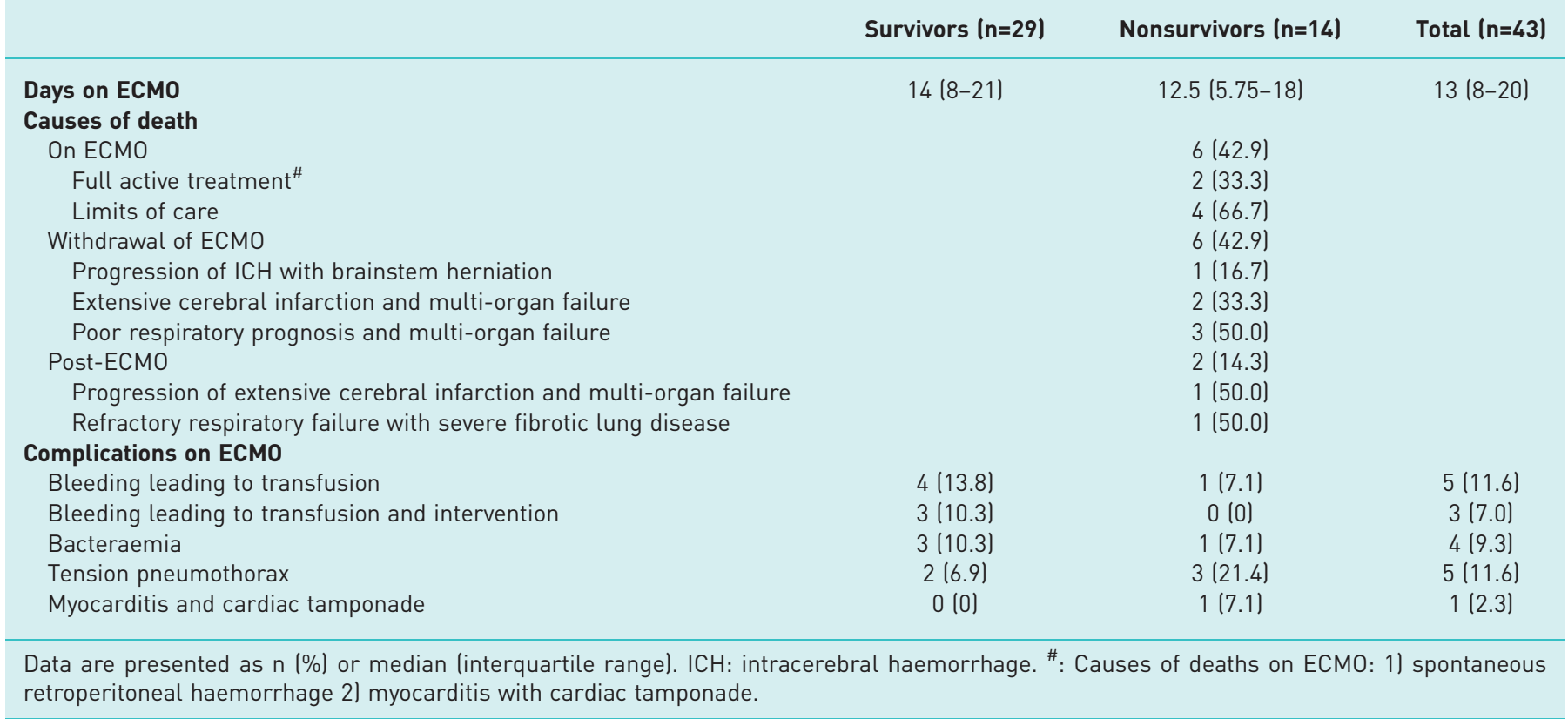

\section{Discussion}

The mortality described in this VV-ECMO series (14 of $43,32.6 \%)$ is lower than in early descriptions. Patients exhibited particular characteristics including poor lung compliance, persistent hyperinflammation and high incidence of thrombosis. Survival in this series is comparable to more recent data from the USA [25] and France [26]. Since the study period, a further 13 patients have completed VV-ECMO at GSTFT, with overall survival to intensive care unit discharge at $71.4 \%$.

The pattern of disease seen in our cohort has been previously described. Exudative lung disease with poor compliance (as described by GATTiNoni et al. [27]), persistent hyperinflammation [28, 29], and increased

\section{TABLE 4 Organ support, therapeutics, other interventions}

\begin{tabular}{|c|c|c|c|}
\hline & Survivors (n=29) & Nonsurvivors (n=14) & Total $(n=43)$ \\
\hline \multicolumn{4}{|l|}{ Organ support } \\
\hline Noradrenaline & 23 (79.3) & $14(100)$ & 37 (86.0) \\
\hline Milrinone & $4(13.8)$ & $5(35.7)$ & $9(20.9)$ \\
\hline Renal replacement therapy & $11(37.9)$ & $8(57.1)$ & $19(44.2)$ \\
\hline Persisting requirement at step-down & $6(20.7)$ & & \\
\hline Anakinra & $7(24.1)$ & $3(21.4)$ & $10(23.3)$ \\
\hline Methylprednisolone $1 \mathrm{~g}$ "pulsed" & $5(17.2)$ & $4(28.6)$ & $9(20.9)$ \\
\hline Hydrocortisone & $1(3.4)$ & $0(0)$ & $0(0)$ \\
\hline \multicolumn{4}{|l|}{ Pharmacological intervention } \\
\hline Remdesivir & $4(13.8)$ & $0(0)$ & $0(0)$ \\
\hline Hydroxychloroquine & $2(6.9)$ & $0(0)$ & $0(0)$ \\
\hline Occlusive thrombus & $1(6.3)$ & $0(0)$ & $1(3.2)$ \\
\hline Nonocclusive thrombus & $12(75.0)$ & $1(100)$ & $13(41.9)$ \\
\hline Mural thrombus only & $3(18.8)$ & $0(0)$ & $3(9.7)$ \\
\hline
\end{tabular}


thrombosis incidence may demonstrate a particular phenotype that defines a later stage of the disease process. Median ferritin and d-dimer seen at ECMO initiation were comparable to values seen after 2 weeks in a cohort of nonsurviving hospitalised patients [30]. A majority of our patients were given immunomodulation [19] after risks of immunosuppressing critically ill patients [31] were weighed against lack of clinical progress and ongoing inflammatory lung insult. Recent trial data showing benefits of dexamethasone in ventilated COVID-19 patients may support the wider use of steroids, although their role in patients on ECMO is unclear [32].

The incidence of PE (27.9\%) was substantially higher than in pre-COVID-19 (9.6\%) in the same centre [33], carrying substantial morbidity in our cohort with RV dysfunction in 50\%. Cannula-related thrombosis rates $(54.8 \%)$ were greater than baseline prevalence [34], and ECMO membrane complication rate was similarly high. Thrombosis risk is a known entity in severe COVID-19 [35], but adjusted anticoagulation targets must be balanced against higher risk of haemorrhagic complication in ECMO [36], the cause of multiple complications and one death in our cohort.

At time of writing, no published literature specifically addresses secondary or coinfection in COVID-19 ECMO. These individuals may represent a distinct cohort microbiologically. The unusual predominance of Klebsiella spp. has been seen elsewhere, as has Candida spp. [37, 38], but remains a focus of further analysis in GSTFT regarding infection control consequences of doubling bedspace usage. Admission procalcitonin was elevated in all patients, but significantly greater procalcitonin in the nonsurvivor group may have limited earlier use of steroids. Re-activation or flares of chronic viral infections including cytomegalovirus must also be considered, especially in those receiving immunomodulation.

Following new commissioning criteria in the UK, the threshold for acceptance of patients onto VV-ECMO has been reinforced by the inclusion of the RESP score. This predictive score is validated in patients already on ECMO [13, 39], but not as a pre-ECMO decision tool. The RESP score was one component of a multi-tool assessment process when deciding which patients should be offered VV-ECMO, and cases with low RESP scores were discussed with a second centre if ECMO was felt to be indicated. Validation of this tool in the UK ECMO population may help to guide future usage.

This series has the inherent limitations of a single-centre study, conducted in a well-resourced and experienced centre, during the early stages of our understanding in a new disease. It is likely that aspects of management will differ over time and between centres, as our understanding of how to treat particular phenotypes improves in any future pandemic waves.

This case series suggests that VV-ECMO, when offered to patients with COVID-19 respiratory failure refractory to conventional ventilatory management, can be associated with a favourable outcome. In COVID-19 patients with severe respiratory failure, early consultation with an ECMO centre and joint decision-making on suitable support modality is a key strategy.

Conflict of interest: None declared.

\section{References}

1 Wang $\mathrm{D}, \mathrm{Hu} \mathrm{B}, \mathrm{Hu} \mathrm{C}$, et al. Clinical characteristics of 138 hospitalized patients with 2019 novel coronavirus-infected pneumonia in Wuhan, China. JAMA 2020; 323: 1061-1069.

2 Yang $\mathrm{X}, \mathrm{Yu} \mathrm{Y}, \mathrm{Xu}$ J, et al. Clinical course and outcomes of critically ill patients with SARS-CoV-2 pneumonia in Wuhan, China: a single-centered, retrospective, observational study. Lancet Respir Med 2020; 8: 475-481.

3 Zheng Y, Sun L, Xu M, et al. Clinical characteristics of 34 COVID-19 patients admitted to intensive care unit in Hangzhou, China. J Zhejiang Univ Sci B 2020; 21: 378-387.

4 Sukhal S, Sethi J, Ganesh M, et al. Extracorporeal membrane oxygenation in severe influenza infection with respiratory failure: a systematic review and meta-analysis. Ann Card Anaesth 2017; 20: 14-21.

5 Noah MA, Peek GJ, Finney SJ, et al. Referral to an extracorporeal membrane oxygenation center and mortality among patients with severe 2009 influenza A(H1N1). JAMA 2011; 306: 1659-1668.

6 Zeng Y, Cai Z, Xianyu Y, et al. Prognosis when using extracorporeal membrane oxygenation (ECMO) for critically ill COVID-19 patients in China: a retrospective case series. Crit Care 2020; 24: 148.

$7 \quad$ Henry BM. COVID-19, ECMO, and lymphopenia: a word of caution. Lancet Respir Med 2020; 8: e24.

8 Henry BM, Lippi G. Poor survival with extracorporeal membrane oxygenation in acute respiratory distress syndrome (ARDS) due to coronavirus disease 2019 (COVID-19): pooled analysis of early reports. J Crit Care 2020; 58: 27-28.

9 NHS England. Extra Corporeal Membrane Oxygenation (ECMO) for Respiratory Failure in adults. 2019.

10 Murray JF, Matthay MA, Luce JM, et al. An expanded definition of the adult respiratory distress syndrome. Am Rev Respir Dis 1988; 138: 720-723.

11 NHS England. Clinical guide for extra corporeal membrane oxygenation (ECMO) for respiratory failure in adults during the coronavirus pandemic. www.england.nhs.uk/coronavirus/wp-content/uploads/sites/52/2020/04/C0156Extra-Corporeal-Membrane-Oxygenation-ECMO-Adult-Speciality-Guide-1.pdf Date last updated: June 25, 2020.

12 Rockwood K. A global clinical measure of fitness and frailty in elderly people. CMAJ 2005; 173: 489-495. 
13 Schmidt M, Bailey M, Sheldrake J, et al. Predicting survival after extracorporeal membrane oxygenation for severe acute respiratory failure. the respiratory extracorporeal membrane oxygenation survival prediction (RESP) score. Am J Respir Crit Care Med 2014; 189: 1374-1382.

14 Sherren PB, Shepherd SJ, Glover GW, et al. Capabilities of a mobile extracorporeal membrane oxygenation service for severe respiratory failure delivered by intensive care specialists. Anaesthesia 2015; 70: 707-714.

15 Camporota L, Caricola EV, Bartolomeo N, et al. Lung recruitability in severe acute respiratory distress syndrome requiring extracorporeal membrane oxygenation. Crit Care Med 2019; 47: 1177-1183.

16 Meduri GU, Headley AS, Golden E, et al. Effect of prolonged methylprednisolone therapy in unresolving acute respiratory distress syndrome. JAMA 1998; 280: 7-8.

17 Meduri GU, Siemieniuk RAC, Ness RA, et al. Prolonged low-dose methylprednisolone treatment is highly effective in reducing duration of mechanical ventilation and mortality in patients with ARDS. J Intensive Care 2018; 6: 53.

18 Waring R. Efficacy and safety of corticosteroids for persistent acute respiratory distress syndrome. $N$ Engl J Med 2006; 14: 1671-1684.

19 Mehta P, McAuley DF, Brown M, et al. COVID-19: consider cytokine storm syndromes and immunosuppression. Lancet 2020; 395: 1033-1034.

20 La Rosée $\mathrm{P}$, Horne $\mathrm{A}$, Hines $\mathrm{M}$, et al. Recommendations for the management of hemophagocytic lymphohistiocytosis in adults. Blood 2019; 133: 2465-2477.

21 Aouba A, Baldolli A, Geffray L, et al. Targeting the inflammatory cascade with anakinra in moderate to severe COVID-19 pneumonia: case series. Ann Rheum Dis 2020; 79: 1381-1382.

22 Dimopoulos G, de Mast Q, Markou N, et al. Favorable anakinra responses in severe Covid-19 patients with secondary hemophagocytic lymphohistiocytosis. Cell Host Microbe 2020; 28: 117-123.

23 Vasques F, Romitti F, Gattinoni L, et al. How I wean patients from veno-venous extra-corporeal membrane oxygenation. Crit Care 2019; 23: 316.

24 Bloomsbury Health. Refer-a-patient ECMO referral portal. Date first accessed: 2nd March 2020. Date last updated: 2nd June 2020. www.signpost.healthcare/ecmo-referral-pathway

25 Osho AA, Moonsamy P, Hibbert KA, et al. Veno-venous extracorporeal membrane oxygenation for respiratory failure in COVID-19 patients: early experience from a major academic medical center in North America. Ann Surg 2020; 272: e75-e78.

26 Falcoz P-E, Monnier A, Puyraveau M, et al. Extracorporeal membrane oxygenation for critically ill patients with COVID-19 related acute respiratory distress syndrome: worth the effort? Am J Respir Crit Care Med 2020; 202: 460-463.

27 Gattinoni L, Chiumello D, Caironi P, et al. COVID-19 pneumonia: different respiratory treatments for different phenotypes? Intensive Care Med 2020; 46: 1099-1102.

28 McGonagle D, Sharif K, O'Regan A, et al. The role of cytokines including interleukin-6 in COVID-19 induced pneumonia and macrophage activation syndrome-like disease. Autoimmun Rev 2020; 19: 102537.

29 Ye Q, Wang B, Mao J. The pathogenesis and treatment of the 'cytokine storm' in COVID-19. J Infect 2020; 80: 607-613.

30 Zhou F, Yu T, Du R, et al. Clinical course and risk factors for mortality of adult inpatients with COVID-19 in Wuhan, China: a retrospective cohort study. Lancet 2020; 395: 1054-1062.

31 Ritchie AI, Singanayagam A. Immunosuppression for hyperinflammation in COVID-19: a double-edged sword? Lancet 2020; 395: 1111 .

32 Chief Investigators of the Randomised Evaluation of COVid-19 thERapY (RECOVERY) Trial on dexamethasone. Low-cost dexamethasone reduces death by up to one third in hospitalised patients with severe respiratory complications of COVID-19. www.recoverytrial.net/news/low-cost-dexamethasone-reduces-death-by-up-to-onethird-in-hospitalised-patients-with-severe-respiratory-complications-of-covid-19 Date last updated: June 16, 2020.

33 Hartley EL, Singh N, Barrett N, et al. Screening pulmonary angiogram and the effect on anticoagulation strategies in severe respiratory failure patients on venovenous extracorporeal membrane oxygenation. $J$ Thromb Haemost 2020; 18: 217-221.

34 Cooper E, Burns J, Retter A, et al. Prevalence of venous thrombosis following venovenous extracorporeal membrane oxygenation in patients with severe respiratory failure. Crit Care Med 2015; 43: e581-e584.

35 CRICS TRIGGERSEP Group (Clinical Research in Intensive Care and Sepsis Trial Group for Global Evaluation and Research in Sepsis), Helms J, Tacquard C, et al. High risk of thrombosis in patients with severe SARS-CoV-2 infection: a multicenter prospective cohort study. Intensive Care Med 2020; 46: 1089-1098.

36 Paden ML, Conrad SA, Rycus PT, et al. Extracorporeal life support organization registry report 2012. ASAIO J 2013; 59: 202-210.

37 Rawson TM, Moore LSP, Zhu N, et al. Bacterial and fungal co-infection in individuals with coronavirus: a rapid review to support COVID-19 antimicrobial prescribing. Clin Infect Dis 2020; in press [https://doi.org/10.1093/cid/ ciaa530].

38 Zhu X, Ge Y, Wu T, et al. Co-infection with respiratory pathogens among COVID-2019 cases. Virus Res 2020; 285: 198005.

39 Brunet J, Valette X, Buklas D, et al. Predicting survival after extracorporeal membrane oxygenation for ARDS: an external validation of RESP and PRESERVE scores. Respir Care 2017; 62: 912-919. 